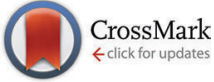

Cite this: J. Mater. Chem. B, 2017, 5, 1227

Received 18th October 2016, Accepted 19th December 2016

DOI: $10.1039 / c 6 t b 02712 a$

www.rsc.org/MaterialsB

\section{Biodegradable porous silk microtubes for tissue vascularization $\dagger$}

\author{
V. E. Bosio, ${ }^{\star a b}$ J. Brown, ${ }^{b}$ M. J. Rodriguez ${ }^{b}$ and D. L. Kaplan ${ }^{b}$
}

Cardiovascular diseases are the leading cause of mortality around the globe, and microvasculature replacements to help stem these diseases are not available. Additionally, some vascular surgeries needing small-diameter vascular grafts present different performance requirements. In this work, silk fibroin scaffolds based on silk/polyethylene oxide blends were developed as microtubes for vasculature needs and for different tissue regeneration times, mechanical properties and structural designs. Systems with 13,14 and $15 \%$ silk alone or blended with 1 or $2 \%$ of polyethylene oxide (PEO) were used to generate porous microtubes by gel spinning. Microtubes with inner diameters (IDs) of 150-300 $\mu \mathrm{m}$ and $100 \mu \mathrm{m}$ wall thicknesses were fabricated. The systems were assessed for porosity, mechanical properties, enzymatic degradability, and in vitro vascular endothelial cell attachment and metabolic activity. After 14 days, all the tubes supported the proliferation of cells and the cell attachment increased with porosity. The silk tubes with PEO had similar crystallinity but a higher elastic modulus compared with the systems without PEO. The silk (13\%)/PEO (1\%) system showed the highest porosity $(20 \mu \mathrm{m}$ pore diameter on average), the highest cell attachment and the fastest degradation profile. There was a good correlation between these parameters with silk concentration and the presence of PEO. The results demonstrate the ability to generate versatile and tunable tubular biomaterials based on silk-PEO blends with potential for microvascular grafts.

\section{Introduction}

Cardiovascular disease (CVD) is the leading cause of mortality around the globe and the number of cases continues to grow. According to the American Heart Association, by 2030, 40.5\% of the US population is projected to have some form of CVD. ${ }^{1}$ Small-diameter blood vessels are key to CVD due to their occlusion or damage, thus the replacement of small-diameter blood vessels (ID $<6 \mathrm{~mm}$ ) with autografts remains the standard clinical approach today. However, considerable morbidity is associated with autologous harvest and scarce availability due to disease or previous organ harvesting. There is growing research work intended on replacing the natural vasculature with such tubing. ${ }^{2-9}$ In the case of synthetic prostheses, polymers like polyethylene terephthalate (PET, Dacron) or expanded polytetrafluoroethylene (ePTFE, Gore-Tex) have been studied, but clinical complications arise, including the regeneration of a non-functional endothelium and the mismatch between the mechanical properties of the grafts and native blood vessels which are key areas of complications. ${ }^{4}$

\footnotetext{
${ }^{a}$ Institute of Applied Biotechnology CINDEFI (CCT La Plata-CONICET, U.N.L.P.), Facultad de Ciencias Exactas, Universidad Nacional de La Plata, Calle 47 y 115 , 1900 La Plata, Argentina. E-mail:vbosio@gmail.com

${ }^{b}$ Department of Biomedical Engineering, Tufts University, Medford, MA 02155, USA

$\dagger$ Electronic supplementary information (ESI) available. See DOI: 10.1039/c6tb02712a
}

To address the above needs, suitable substitutes for smalldiameter grafts are needed. Bioprinting strategies, for example, have demonstrated their potential use to pattern cells or fabricate different tissues and organs including blood vessels or cardiac patches. However, these approaches still have technical challenges in terms of vascularization, among others. ${ }^{10-14}$ Mimicking natural vascular architecture and rebuilding microvascular networks remain a challenge. ${ }^{5}$ To develop functional and appropriate structures for these small-diameter vessels, key properties like biocompatibility to promote tissue regeneration (non-toxic/immunogenic/thrombogenic or susceptible to infections and a functional endothelium); mechanical properties, similar to native vessels (compliance, burst pressure, compression resistance to aneurysms); and processability (low manufacturing costs, readily available with a large variety of lengths and diameters, sterilizable, storability) are needed., ${ }^{45}$

Vascular tissue engineering approaches include different strategies to emulate the natural function of the small-diameter vessels including cell-based strategies (with prevascularization or neoangiogenesis (induction in vitro) associated processes) and scaffold-based strategies (biological scaffolds (acellular matrix) or synthetic scaffolds (from synthetic or biopolymers)). Even when decelullarized structures satisfy many of the requirements, one major limitation of the most animal-derived matrices is that they lack standardization and reproducibility. ${ }^{16}$ The use of synthetic 
scaffolds offers advantages like consistency in materials and fabrication techniques, reproducible standardized design and high volume production, and the avoidance of the use of animal sources. However, these systems often suffer from challenges in cell interactions or thrombolytic responses.

Since different histological structures and mechanical functions are related to each region of the circulatory system, the fabrication of scaffolds suitable for these tissue needs requires tunable features to meet this range in vessel requirements. Therefore, materials with tunable properties are an advantage. Different degradation rates, for example, could be relevant in terms of repairs in different zones or in response to different degrees of damage. The porosity for the delivery of oxygen and nutrients relates to applications where high transport rates, but not mechanical strength, are needed versus low porosity tubes where mechanical integrity, but not transport, is critical. The rate of tissue ingrowth of grafts can be improved through optimizing the porosity or the permeability of the grafts. For synthetic grafts prepared from ePTEF, an intermodal distance of $30 \mu \mathrm{m}$ was better than distances of 50 and $100 \mu \mathrm{m}$ when tested in humans. ${ }^{17}$

The ability to construct complex geometries and small dimensions, the mechanical properties, and the need for biofunctionalization present additional challenges. Some of these deficiencies can be addressed with biopolymers due to their biocompatibility, biodegradability, minimal inflammatory reactions and excellent mechanical properties. For example, silk-based materials have been used in tissue regeneration such as for bone, cartilage, blood vessels, skin and nerves. ${ }^{18-25}$ The structures generated based on silk derived from Bombyx mori are easy to store and sterilize, and, depending on the selected technique, can be fabricated into scaffolds such as vessels with small dimensions with tunable geometries. ${ }^{26}$ Additionally, drug delivery properties of silk provide a useful option to build structures carrying specific therapeutic molecules, ${ }^{27,28}$ including pro-angiogenic growth factors, to promote the natural development of microvasculature once the graft is in the body, helping the proliferation of resident vascular progenitor cells.

The generation of microtubes based on silk has been reported by our lab previously. ${ }^{29}$ However, further studies are necessary to regulate or tune the features of these structures. Thus the focus of this study was to further refine the relationships between the processing and degradation of materials, mechanical properties and porosity of silk tubes in the context of vascular tissue needs. Compared to dip-coated silk microtubes, the gel-spinning approach based on silk/polyethylene oxide blends developed in this study showed tunable degradation behavior, various mechanical properties, better structural homogeneity and good cell growth in vitro, implying feasibility for different microvascular tissue requirements.

\section{Experimental}

\section{Preparation of aqueous silk solutions}

A $5-6 \%(\mathrm{w} / \mathrm{v})$ silk fibroin (hereafter termed silk) aqueous solution was obtained from B. mori silkworm cocoons using previously described procedures. ${ }^{30-35}$ Briefly, the silkworm cocoons (supplied by Tajima Shoji Co., Ltd, Yokohama, Japan) were extracted in $0.02 \mathrm{M}$ sodium carbonate solution by boiling for 5, 10, 15 or 20 minutes, rinsed with distilled water, dissolved in 9.3 $\mathrm{M}$ lithium bromide, and dialyzed against distilled water using a cellulose dialysis tubing (molecular weight cutoff, MWCO, 3500, Fisherbrand by Fisher Scientific, Pittsburgh, PA) for $48 \mathrm{~h}$. The resulting $5-6 \%(\mathrm{w} / \mathrm{v})$ silk solution was then concentrated using a CentriVap Vacuum Concentrator (Labconco, Kansas City, MO) to produce a $7-15 \%$ (w/v) silk aqueous solution. All silk solutions were stored at $4{ }^{\circ} \mathrm{C}$ until the concentration process.

\section{Winding device and winding of silk-based tubes}

A custom silk spinning device (SolidWorks, Concord, MA) with a spinning mandrel driven by a two-axis stepper motor (Haydon Switch \& Instrument, Waterbury, CT) that drove the mandrel both axially and rotationally was used to make the microtubes as described previously. ${ }^{36}$ The rotational speed was $200 \mathrm{rpm}$ while the axial slew rate (ASR) was $2 \mathrm{~mm} \mathrm{~s}^{-1}$ over the maximum $(5 \mathrm{~cm})$ stroke length. A Teflon-coated stainless steel rod of $400 \mu \mathrm{m}$ diameter (McMaster-Carr, Atlanta, GA) was used as the silk spinning mandrel and was fixed to the motor shaft with a coupling adapter. Teflon guide blocks, allowing the ends of the rod to slide freely, stabilized the rod. For silk blend deposition, a three-axis manipulator was used to precisely position the syringe needle. ${ }^{36}$

Tubes were prepared by pushing $10-15 \%(\mathrm{w} / \mathrm{v})$ silk solution through a 27 or 29 gauge needle onto a rotating and axially reciprocating mandrel. After evenly coating with concentrated silk, the transformation from amorphous liquid to the betasheet crystalline form was induced by treatment with methanol. ${ }^{37}$ The silk-coated mandrels were placed at $-25{ }^{\circ} \mathrm{C}$ for 6 hours and then dried with two lyophilization cycles. After MetOH treatment, the samples were washed overnight if polyethylene oxide (PEO) was present or for 2 hours in the absence of PEO. All the samples were placed in water to remove the silk tubes from the Teflon-coated stainless steel rods. Prior to any cell-based experiments, the microtubes were air-dried and sterilized using ethylene oxide (ETO). The samples were placed in sterilization pouches and exposed to ETO gas for $10 \mathrm{~h}$, followed by $2 \mathrm{~h}$ of degassing in the sterilizer chamber and an additional $24 \mathrm{~h}$ of degassing in a vacuum oven at room temperature to remove residual ETO.

\section{Preparation of porous silk/PEO microtubes}

Concentrated silk solutions were blended with different volumes of $5 \mathrm{wt} \% \mathrm{PEO}^{29}$ to form ratios of silk/PEO of 100/0, 99/1 and 98/2 (wt\%). The silk/PEO blends were gently mixed at $37{ }^{\circ} \mathrm{C}$ using a spatula. Silk and silk/PEO microtubes were prepared using the same gel-spinning technique as described above, and then frozen and dried overnight. After drying, the microtubes were treated with methanol and immersed in distilled water overnight at room temperature $\left(24 \mathrm{~h}, 25^{\circ} \mathrm{C}\right)$, thus facilitating the extraction of the PEO phase and leaving porous microtubes. 


\section{Imaging}

The morphology of the silk tubes was observed by scanning electron microscopy (SEM) (a Zeiss EVO MA10 SEM equipped with an SE1 detector, Carl Zeiss, Oberkochen, Germany) at $3.0 \mathrm{kV}$. Fractured sections of the silk-based tubes were obtained from wet tubes using surgical scissors and dried overnight. Samples were mounted on a copper plate and sputter-coated with a $20-30 \mathrm{~nm}$ thick $\mathrm{Au} / \mathrm{Pd}$ layer prior to imaging. The SEM images, along with image analysis software (ImageJ, National Institutes of Health, USA), were used to determine wall thickness and pore size distribution. For pore size distribution, 300 measures over 3 images were analyzed for each pore size study. HUVECs present in the microtubes after 3 and 14 days were observed by optical microscopy (LCS Lite, Leica Microsystems, Mannheim/ Wetzlar, Germany) and stained using Calcein AM/rhodamine dyes according to company protocols (Invitrogen, Carlsbad, CA) for live/dead imaging. Image intensities were measured using a Leica fluorescence microscope (LCS Lite, Leica Microsystems, Mannheim/Wetzlar, Germany).

\section{Mechanical evaluation}

Tensile tests were performed on hydrated silk strips of $2-9 \mathrm{~mm}$ width and 20-30 mm length and fabricated by the same gelspinning technique used to make the tubes but with a $10 \mathrm{~mm}$ diameter spinning mandrel. The testing was performed using an Instron 3366 testing frame (Instron, Norwood, MA) with 5 and $10 \mathrm{~N}$ capacity load cells and BioPuls Bath, Submersible Pneumatic Grips (Instron BioPlus Systems Solutions for Biomedical Testing, Norwood, MA) under physiological conditions. This characterization was performed for a range of silk-based formulations to determine the difference in properties as a function of the extraction boiling time (during the degumming process) and the additive. Sample tubes $(N=3$ each tube type) were hydrated in $0.1 \mathrm{M}$ phosphate-buffered saline (PBS) for 24 hours before clamping and submerging in a temperature-controlled BioPuls Bath $\left(37 \pm 0.3{ }^{\circ} \mathrm{C}\right)$ filled with PBS for at least 5 min prior to testing. The initial length of each strip was obtained using a caliper. Small parts of each strip to be tested were stored and cross sections were prepared for histology. Hematoxylin and eosin staining were performed to increase contrast in the cross sections. Images of slides were taken using an optical microscope (Axiovert S100, Zeiss, Germany) equipped with a digital imaging video camera (DXC-390, Sony, Tokyo, Japan). The cross-sectional areas were calculated using Image from the $\mathrm{NIH}^{38}{ }^{3}$ These cross-sectional areas were used to calculate stress from the measured force. Samples were prepared by placing two dots axially for video axial measurements (Fig. 1). A crosshead displacement rate of $0.01 \%$ per min was used, and video axial strains were measured using video extensometer software.

Stress and strain were computed from the measured force and displacement using Bluehill's Instron software according to cross-sectional area measurements. The linear elastic modulus was calculated using a least-squares fitting between $0.02 \mathrm{~N}$ load and $3 \%$ strain past that point. The yield strength was determined by offsetting this least-squares line by $5 \%$ strain and marking the
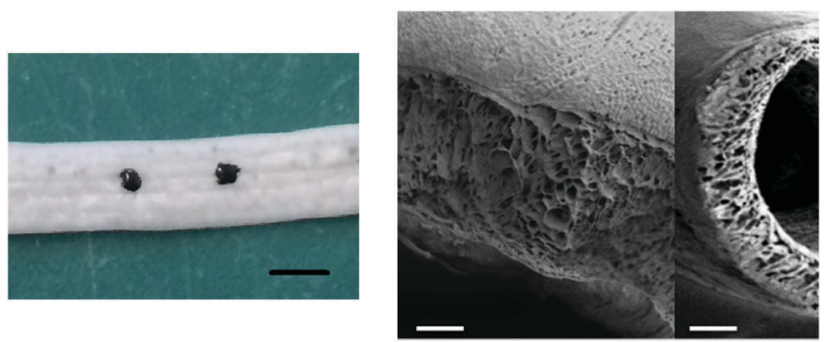

Fig. 1 Silk strip (2-9 $\mathrm{mm}$ width and $20-30 \mathrm{~mm}$ length) fabricated with a $10-\mathrm{mm}$ diameter spinning mandrel. Samples were prepared by placing two dots axially for video axial measurements. Scale bar $=5 \mathrm{~mm}$. Cross-sectional SEM images of silk strips (left) and microtubes (right). Scale bar $=100 \mu \mathrm{m}$.

data intercept. Ultimate tensile strength (UTS) was determined as the highest stress value attained during the test.

\section{Characterization of silk-based tubes}

The infrared spectra of the silk and silk/PEO microtubes were measured using a JASCO FT-IR 6200 spectrometer (JASCO, Tokyo, Japan). Microtubes were dehydrated in an airflow hood to minimize the contribution of water peak during FT-IR analysis, and evaluated with each spectrum acquired over the range of $4000-400 \mathrm{~cm}^{-1}$ for 66 scans with a resolution of $4 \mathrm{~cm}^{-1}$. The secondary structure of silk was evaluated by Fourier self-deconvolution (FSD) of the FTIR spectra covering the amide I region using Opus 5.0 software. ${ }^{38}$

Pore size distribution was calculated using SEM images, along with image analysis software (ImageJ, the National Institutes of Health, USA). A total of 300 measures were carried out over 3 images for each pore size study, where mean was indicated for each system as well as standard deviation (SD) values.

Silk-based microtubes were assessed for enzymatic degradation over time using a protocol described previously. ${ }^{39}$ Briefly, mass loss induced by proteolytic degradation was determined by incubating hydrogels in protease XIV (5.3 units per mg, SigmaAldrich, St. Louis, MO, reconstituted in water by stirring for one hour). The mass loss was calculated at each time point as weight loss divided by initial microtube mass during 10 days (1).

$$
\text { Weight loss } \%=\left(\left(W_{0}-W_{t}\right) / W_{0}\right) \times 100
$$

Silk-based microtubes $(1 \mathrm{~cm}$ in length, $N=3$ per group and time point) were incubated in $0.5 \mathrm{~mL}$ of $0.1 \mathrm{mg} \mathrm{mL}^{-1}$ protease XIV in water at $37^{\circ} \mathrm{C}$ for a period of 21 days. As negative controls, silkbased microtubes were incubated in water instead of enzymatic solution. ${ }^{39}$ Enzyme solution was removed and replaced with fresh enzyme once every 24 hours for up to 21 days. At each time point, samples were removed from incubation and washed with deionized water to remove adsorbed protein. Microtubes were then air-dried and weighed. For the comparison of the initial weight, the samples were initially incubated in deionized water for 24 hours at room temperature to remove any soluble material.

\section{Cell culture}

Human umbilical vein endothelial cells (HUVECs) (Cambrex, Walkersville, MD) were used to seed the tubes. Prior to seeding, 
HUVECs were cultured according to previously reported protocols in optimized growth media (EGM-2) consisting of Endothelial Basal Medium-2 (Lonza, Walkersville, MD) supplemented with $100 \mathrm{U} \mathrm{mL}^{-1}$ penicillin, $1000 \mathrm{U} \mathrm{mL}^{-1}$ streptomycin, and $0.2 \%$ fungizone antimycotic (GIBCO, Carlsbad, CA). Cells were cultured at $37{ }^{\circ} \mathrm{C}, 5 \% \mathrm{CO}_{2} / 95 \%$ air, and $95 \%$ relative humidity.

Cell culture medium was replenished twice per week, and cells were passaged four times at approximately $80 \%$ confluence using trypsin-EDTA ( $0.25 \%$ trypsin with $1 \mathrm{mM}$ EDTA- $4 \mathrm{Na})$ before being used to seed the scaffolds.

Silk-based tubes were sterilized using ETO and prior to cell seeding, microtubes of $5 \mathrm{~mm}$ length were presoaked in media overnight at $37{ }^{\circ} \mathrm{C}\left(5 \% \mathrm{CO}_{2} / 95 \%\right.$ relative humidity) in 48 well plates. Media were then aspirated from scaffolds the following day. Microtubes were pre-coated with $10 \mathrm{mg} \mathrm{mL}^{-1}$ of collagen solution and incubated for 30 minutes at $37{ }^{\circ} \mathrm{C}$ in order to improve the attachment outcome (most likely due to the presence of Arg-Gly-Asp tripeptide (RGD sequences) available for cell binding $)^{40}$ and reduce the times for in vitro studies. The excess collagen was removed by washing with PBS buffer, and $7 \mu \mathrm{L}$ of cell pre-cultured suspensions were pipetted onto the scaffolds to reach $2 \times 10^{5}$ cells. Cells were allowed to attach for $1.5 \mathrm{~h}$ at $37{ }^{\circ} \mathrm{C}\left(5 \% \mathrm{CO}_{2} / 95 \% \mathrm{RH}\right)$, and $200 \mu \mathrm{L}$ of media was then added to each scaffold (one scaffold/well of 48-well plate) and replenished every 4 days. ${ }^{41}$

\section{Metabolic activity}

To assess changes and differences in cellular activity, the different microtubes were assayed every 3 days using Alamar Blue (Invitrogen Corp, Grand Island, NY). Briefly, a 9:1 (v/v) dilution of Alamar Blue to cell culture medium was added directly to the samples and then incubated for 4 hours. The following incubation fluorescence readings were taken using a multimode multiplate reader (SpectraMax M2, Molecular devices, Sunnyvale, CA) at $530 \mathrm{~nm}$ excitation and $590 \mathrm{~nm}$ emission using SoftMax Pro software. Arbitrary units were determined as relative units of fluorescence intensity from the reduction of resazurin found in Alamar Blue to red fluorescent resorufin in the presence of metabolically active cells.

The same samples were measured at each time point (sample size per group per time point was $n=3$ ). Graphs were normalized to the fluorescence reading of the Alamar Blue solution from the non-seeded scaffolds.

\section{Statistical analysis}

All the experiments were independently replicated with a minimum sample size of $N=3$ or $N=300$ for pore size measures. Values were expressed as average (or mean and mode for pore sizes) plus or minus the standard deviation $( \pm \mathrm{SD})$. Student's $t$-test was performed for paired observations. A value of $p<0.05$ was considered statistically significant.

\section{Results and discussion}

\section{Silk-based microtube fabrication}

Silk-based tubes were previously generated using a gel-spinning process with concentrated silk on a stainless steel rod of 400 micron diameter, using methanol to induce $\beta$-sheet formation which provided both stability in aqueous solution as well as improved mechanical properties. ${ }^{37}$ This technique provided solid silk tubes with an ID of about 150-300 micrometers and a wall thickness of 100 micrometers. To improve the diffusion properties of silk microtubes, porous tubes were generated by adding 1 and 2 percent of PEO to the concentrated silk. By adding a specific weight percent of PEO and using different aqueous silk solution percentages, pores of defined size were obtained. The microtubes were analyzed for pore size distribution and roughness at the exterior surface as well as at the cross section of the microtube using SEM.

As shown in Fig. 2, pore size distributions in the silk-based microtubes were controlled by changing the concentration of silk as well as by the presence of PEO. ${ }^{29}$ No difference was found between 1 and 2 percent of PEO in terms of porosity (data not shown). Porosity was enhanced with lower silk concentrations in silk/PEO blends (Table 1 and Fig. S1 of the ESI $\dagger$ ). The surfactant properties and hydrophilicity of PEO promoted the porous structures observed in the silk/PEO systems. The water extraction step after lyophilization of the tubes helped to leach out PEO to increase the porosity compared with the silk-only systems. ${ }^{42}$ Topography analysis using Image J was also performed (see Fig. S2 of the ESI $\dagger$ ).

The presence of beta-sheet in all formulations of silk and silk/PEO microtubes was confirmed using FT-IR (Fig. 3). Each microtube demonstrated a predominant $\beta$-sheet structure $(1697$, $1623 \mathrm{~cm}^{-1}$, amide I; $1514 \mathrm{~cm}^{-1}$, amide II), which was induced by the methanol treatment applied to all microtubes. Methanol treatment did not enhance the $\beta$-sheet structure observed by FT-IR when PEO was present, and there were no differences in the amide I and amide II spectral bands between microtubes of pure silk and those of silk/PEO blends (Fig. 3 and Fig. S3, S4 of the ESI $\dagger$ ), agreeing with previously reported structural data using silk and PEO films. ${ }^{42}$

\section{Mechanical properties}

The study of strain-stress curves for silk/PEO microtubes in the initial longitudinal tensile test showed a Young's modulus as a function of silk concentration and porosities (Fig. 4).

The uniaxial tensile properties of native human blood vessels are presented in Table 2. Even with some discrepancies in the literature among the reported values of various mechanical properties for the same tissue by different researchers (due to differences in methods), ${ }^{43}$ the comparison of elasticity and ultimate tensile strength among mean values of different material grafts (natural vessels, ${ }^{4-46}$ previously developed silk microtubes ${ }^{47}$ and our systems) showed an improvement in the mechanical properties with respect to native blood vessels in terms of ultimate tensile strength (Table 2). These systems showed average values of UTS of $0.56 \mathrm{MPa}$, and while they are smaller caliber vessels than saphenous veins $(0.5 \mathrm{~mm}-0.5 \mathrm{~cm})$ or coronary vessels $(0.1-10 \mathrm{~mm})$, it is worth noting that these values are in the order of those of human saphenous veins, the current gold standard for bypass grafts even of small diameter vessels $(<6 \mathrm{~mm}),{ }^{48}$ and are also higher than our previously reported silk tubes indicating feasibility for future in vivo 


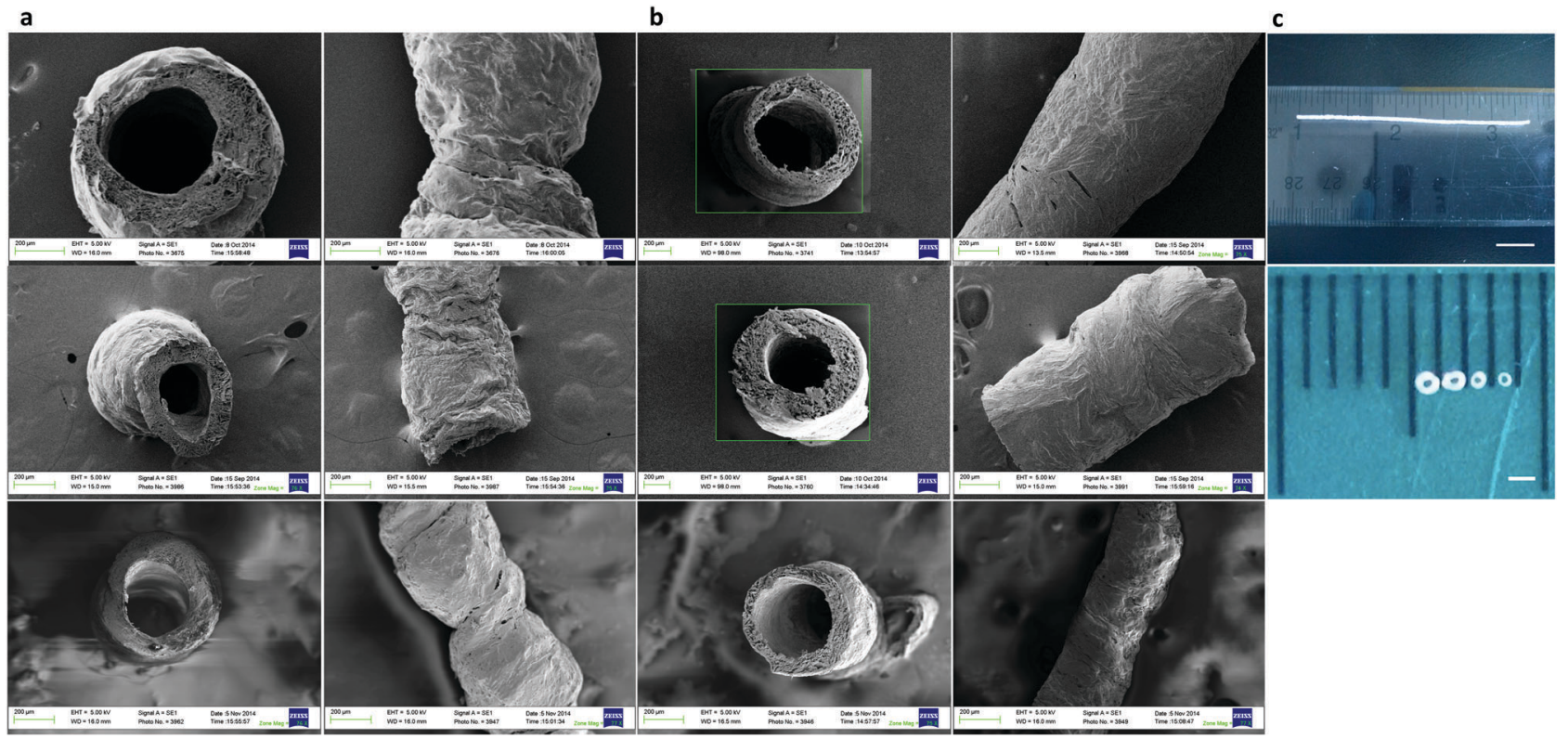

Fig. 2 Microscopy and SEM images for cross section and length of silk and silk/PEO microtubes. (a) Top to bottom: 13, 14 and 15\% silk microtubes; (b) Top to bottom: Blends of PEO at 1\% and silk 13, 14 and 15\% microtubes; (c) Top to bottom: One silk microtube (scale bar $=1 \mathrm{~cm}$ ) and different cross sections of silk and silk/PEO tubes (scale bar $=1 \mathrm{~mm}$ ).

Table 1 Porosity analysis using ImageJ for silk and silk/PEO blends. Table comparing all data (SD = standard deviation)

\begin{tabular}{llllllll}
\hline & Silk & \multicolumn{5}{c}{ Silk/PEO } \\
\cline { 2 - 3 } Pore size $(\mu \mathrm{m})$ & $13 \%$ & $14 \%$ & $15 \%$ & & $13 \%$ & $14 \%$ & $15 \%$ \\
\hline Mean & 9.61 & 7.46 & 4.15 & & 20.01 & 16.96 & 7.59 \\
Mode & 5.43 & 4.19 & 2.19 & & 12.45 & 13.49 & 5.03 \\
SD & 4.27 & 4.31 & 2.61 & & 8.38 & 6.14 & 3.23
\end{tabular}

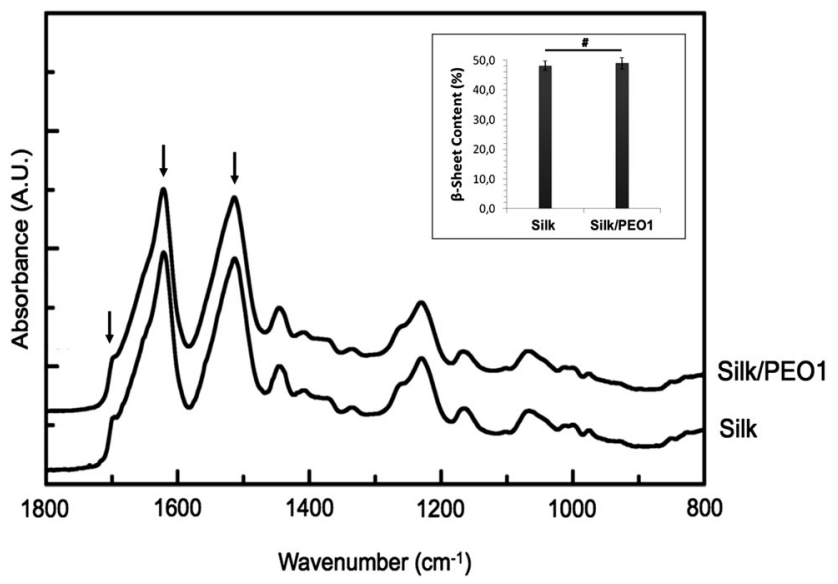

Fig. 3 FT-IR spectra for silk and silk/PEO1 blends and quantification of $\beta$-sheet content of silk and silk/PEO1 blends. (\#) Not statistically significant $(P<0.05)$.

studies involving tubes manufactured with silk/PEO blends. In general, these new generation silk/PEO systems showed a smaller inner diameter compared with previous work, without losing the mechanical properties and with a non-thrombogenic shape, even for low silk concentrations.

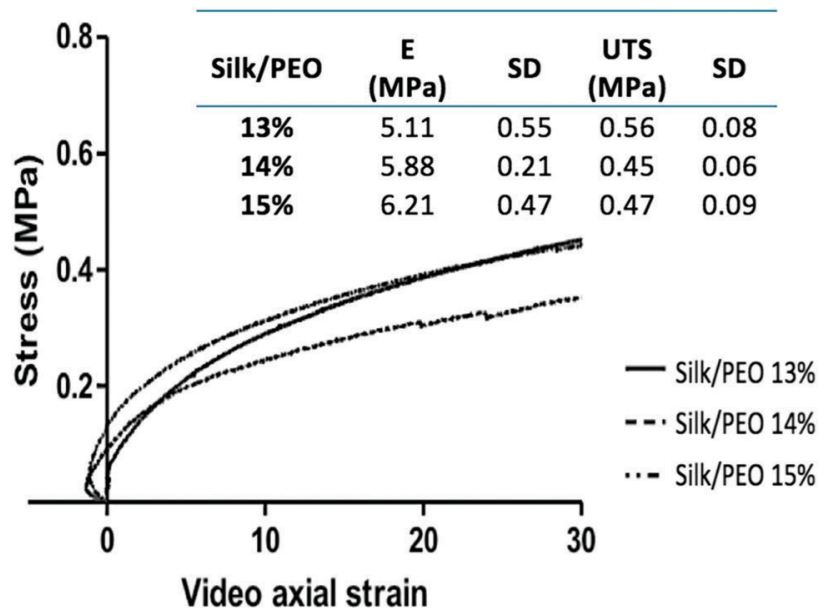

Fig. 4 Strain-stress curves for samples in initial longitudinal tensile tests. Average Young's modulus (E) was calculated between 0 and 5 video axial strain percentages. Average tensile stress at ultimate tensile strength (UTS) was calculated from tensile stress vs. video axial strain plots (SD = standard deviation).

Table 2 Comparison of the mechanical properties of native aorta and graft material

\begin{tabular}{lll}
\hline Material graft & Elastic modulus (MPa) & $\begin{array}{l}\text { Ultimate tensile } \\
\text { strength (MPa) }\end{array}$ \\
\hline Saphenous vein & $1.45 \pm 0.19$ to $23.7 \pm 15$ & $0.95 \pm 0.11$ \\
Coronary vessels & 0.81 to $1.72 \pm 0.26$ & 0.51 to $1.55 \pm 0.87$ \\
Silk tubes $^{\text {Silk/PEO tubes }}{ }^{a}$ & $2.20 \pm 0.90$ & $0.27 \pm 0.11$ \\
& $5.11 \pm 0.59$ & $0.56 \pm 0.11$
\end{tabular}

${ }^{a}$ Specimens of silk $13 \%$ PEO 1. 


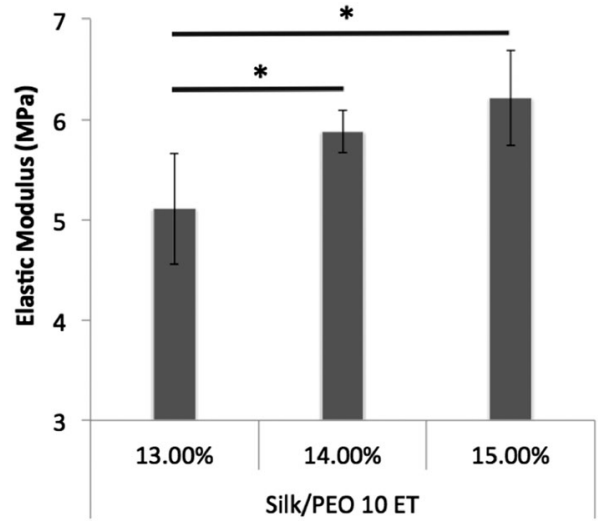

Fig. 5 Elastic modulus for silk/PEO systems. The samples are referred to as $10 \mathrm{ET}$, where "ET" stands for extraction time in minutes. (*) Statistically significant $P<0.05$.

The presence of PEO in the formulations enhanced the Young's modulus (Fig. 5). These silk/PEO solution blends reduced the material's brittleness and enabled processing and handling of the final material, providing better conditions for further processing ${ }^{30,31,49}$ including gel spinning.

Despite a similar $\beta$-sheet content in samples up to 60 minutes extraction time (ET) and the same methanol treatment, ${ }^{50}$ the samples with PEO (10 ET) had different elastic moduli (Fig. 5) and rheological properties determined by tensile stress and viscosity (data not shown). The enhancement of $E$ was observed for different silk concentrations in the silk/PEO systems, when PEO was present compared with only silk microtubes, or when the extraction time was increased (data not shown). This feature can be an advantage to manipulate the material without changing other properties related to the degumming process. ${ }^{50}$

\section{Enzymatic degradation}

The microtubes were subjected to in vitro proteolytic digestion using protease XIV based on our previous results. ${ }^{39}$ Degradation was observed in all silk samples incubated in the protease solution, demonstrating a linear degradation rate in terms of weight ratio (Fig. 6). At each time point, silk microtubes became more brittle, thereby undergoing degradation. After 14 days a decrease in degradation was observed in all non-PEO systems, and microtubes made with $15 \%$ silk solution showed integrity until the last time point. The degradation of the silk/PEO microtubes showed a higher degradation rate than the silk-only tubes. The $13 \%$ silk/PEO systems showed a significant difference from the systems, with the highest degradation rate. No significant differences were found between the 1 and $2 \%$ of PEO for all the systems. Control samples incubated in water but without enzyme did not exhibit any mass loss.

The microtubes demonstrated varying biodegradation profiles based on silk concentration and the presence of PEO. This tunable degradation profile is useful in terms of achieving different tube properties.

\section{Vascular cell attachment and proliferation}

The attachment and proliferation of endothelial cells in vitro in the tubes were assessed by coating with endothelial cells.

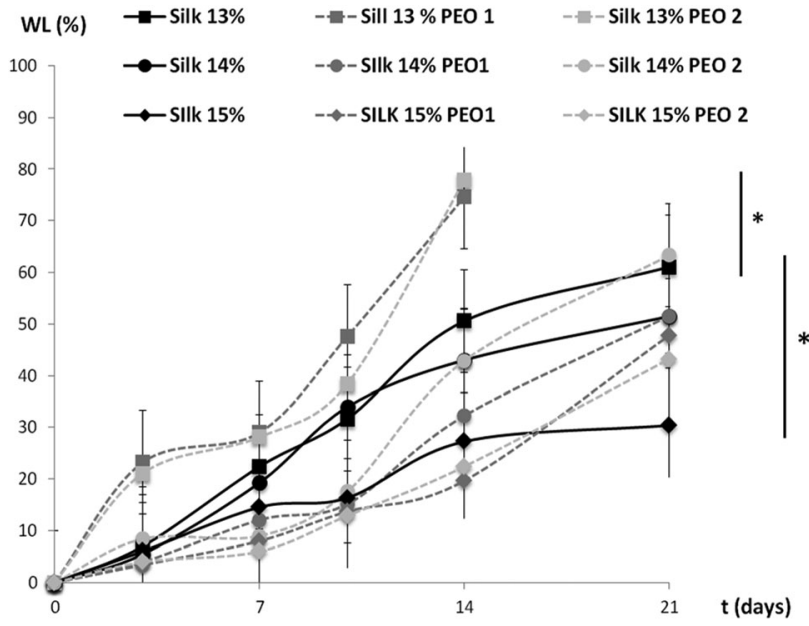

Fig. 6 Enzymatic degradation. Normalized degradation profiles as a percentage of weight loss $(\mathrm{WL})$ vs. time $(t)$ for silk (different concentrations: $13,14$ and $15 \%)$ and blends of silk (13, 14 and 15\%) and PEO ( 1 and $2 \%)$. (*) Statistically significant $P<0.05$.

The ability of these cells to grow on silk-based tubes in vitro was followed by optical and fluorescence microscopy.

As shown in Fig. 7, more cells attached to the silk/PEO microtubes than the silk-only systems at the same concentrations. Comparing the silk/PEO systems, better attachment was observed with higher porosity. This suggests higher potential for the formation of an endothelial cell lining on the inner surface of graft lumens for low concentrations of silk/PEO systems. a

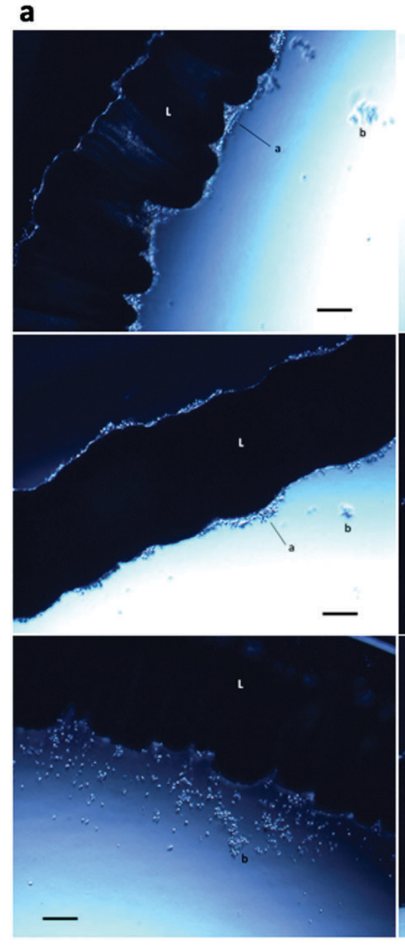

b

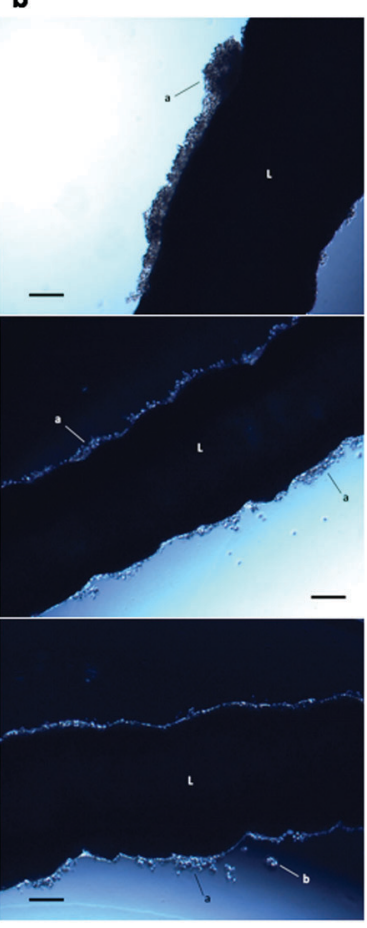

Fig. 7 Optical microcopy images $(20 \times)$ for HUVECs cultured on the microtubes. (a) Top to bottom: Only silk of 13, 14 and 15\% (w/v) (b) top to bottom: PEO $1 \%$ and silk of 13,14 and $15 \%(w / v)$. In all images, scale bars $=100 \mu \mathrm{m}$; $\mathrm{L}=$ microtube lumen; $\mathrm{a}=$ attached cells; $\mathrm{b}=$ non-attached cells. 


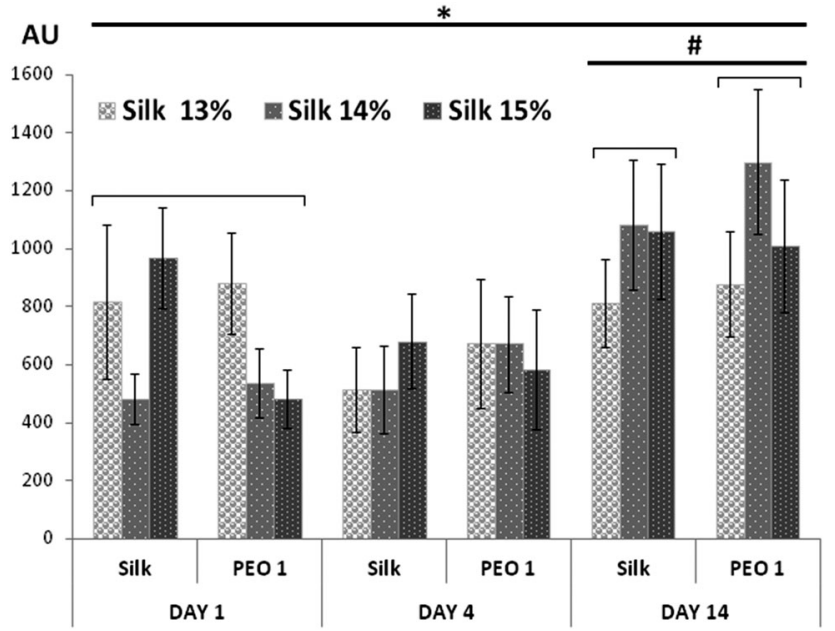

Fig. 8 Cellular activity. Normalized metabolic activity graphed as fluorescence intensity in arbitrary units (AU) vs. time for all the systems: (a) silk controls for different concentrations and (b) silk/PEO (1\%) blends. ${ }^{*}$, \#) Statistically significant $(P<0.05)$ and not statistically significant, respectively.

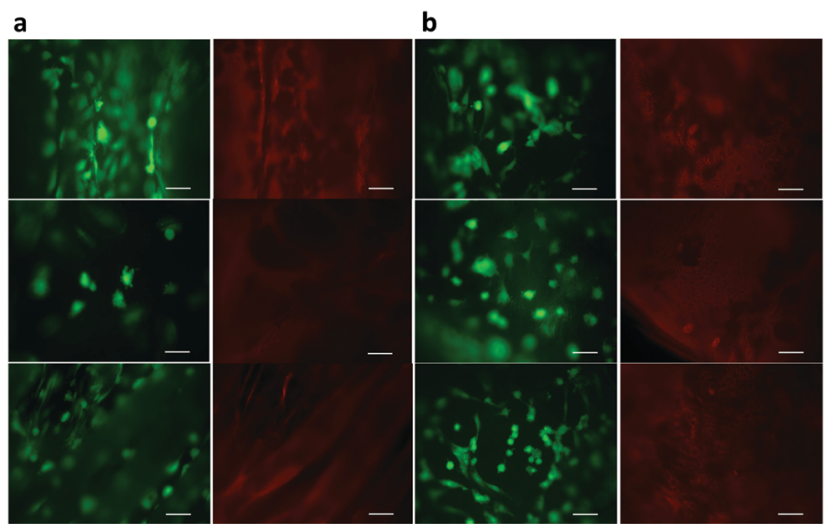

Fig. 9 Live/dead study of HUVECs cultured on half-moon shaped microtubes stained for the study of 14 day cultures. (a) Top to bottom: Silk of 13 , 14 and $15 \%(\mathrm{w} / \mathrm{v})(\mathrm{b})$ top to bottom: PEO $1 \%$ and silk of 13,14 and $15 \%(\mathrm{w} / \mathrm{v})$ Calcein AM staining (left, green) shows live cells attached on the microtubes, and rhodamine staining (right, red) shows the microtube matrix supporting the cells. Scale bars $=100 \mu \mathrm{m}$.

Despite the optical microscopy attachment observed, the ALB profiles showed a decrease in cell viability after 4 days for some structures (silk 13\%, silk 13\%/PEO and silk 15\%), while the other systems showed similar levels. With poor attachment to increase the cellular mass on the structures, the transfer steps during the procedure could affect the final cell amounts to react with $\mathrm{ALB}$ and be responsible of the cell viability levels.

However, after 14 days, all the systems showed a significant increase in cell activity detected in spite of the possible losses of cell mass before the readings, evidence of a stronger adhesion to the microtubes, suggesting that the graft materials can provide cell attachment and infiltration necessary for remodeling (Fig. 8).

The proliferation and cell adhesion were monitored by fluorescence microscopy for calcein AM versus rhodamine-stained
HUVECs in a live/dead study. The images showed that after 14 days, cells were actively proliferating (Fig. 9).

\section{Conclusions}

Microtubes (150-300 $\mu \mathrm{m}$ ID) of silk/PEO blends may offer a novel tunable system for microvascular grafts. These specific blends of silk/PEO (13, 14 and $15 \%$ of aqueous 10 ET silk solutions with 1 and $2 \%$ of PEO) allowed the fabrication of homogeneous and reproducible tubular scaffolds with tunable mechanical properties and controlled degradation behavior using an aqueous, simple and reproducible method like gel spinning. The use of 10 ET silk allowed its utility with lower silk concentrations to enhance results over previous studies, including our reported systems by dip coating and gel spinning methods. ${ }^{28,36,42,47}$ These blends showed different profiles of degradation and porosity for the microtubes, without losing their structural integrity, crystallinity or elasticity but achieved patency and smaller external/IDs.

The presence of high viscosity PEO allowed us to work with lower silk concentrations and avoid complicated procedures related to manipulating the material during and after the gel spinning process. As a result, the modulation of tube structures was possible including avoiding defects during spinning to decrease the possible zones of platelet adhesion to preserve non-thrombogenic features.

The porous silk-based microtubes were characterized in terms of pore size, crystalline structure, mechanical properties, enzymatic degradation, cell attachment and metabolic activity. Porous silk microtubes could be produced with good control over many properties. The presence of PEO increased the porosity and elasticity in all the systems. Many reports have suggested the need for improving the porosity and permeability of the scaffolds to optimize tissue ingrowth for vascular grafts, ${ }^{7,17,51,52}$ and these porous silk-based microtubes should be able to address this need. With $13 \%$ silk in the blends, higher porosity and pores of $20 \mu \mathrm{m}$ average diameter (in the size range for better ingrowth tissue rates in humans) were found accompanied by higher cell attachment and degradability, the parameters that can improve ingrowth as well. These systems showed an UTS of $0.56 \mathrm{MPa}$, on the order of human saphenous veins, the current gold standard for bypass grafts even of smalldiameter vessels ( $<6 \mathrm{~mm}$ ), indicating feasibility for future in vivo studies involving tubes prepared with silk/PEO blends. In general, these new-generation silk/PEO systems showed a smaller inner diameter compared with previous work, without losing the mechanical properties and with non-thrombogenic surfaces, even for low silk concentrations, presenting a promising material for microvasculature replacement. Even when these materials are compared with human vessels that have larger inner diameters, they look similar in terms of porosity, while degradation and remodeling rates will need to be assessed in vivo. The ability of these silk scaffolds to support vascular cell adhesion that could remodel the graft material should also be useful. These microtubes could be included within other kinds of scaffolds to enable the pre-vascularization process and to build 
natural connections with existing vasculature by anastomose processes to avoid graft problems. ${ }^{53}$

The tunable profile of degradation for silk scaffolds may offer alternatives to replace microvascular tissues under different conditions. While these initial results are promising, the relation between silk scaffold degradation and graft remodeling rates will need to be assessed in vivo. The addition of therapeutic drugs in the silk/PEO blends, the inhibition of complement activation, oxidative stress, and thrombosis pathways may represent additional variants to pursue with the silk tube systems as potential therapeutic targets for promoting microvascular health. ${ }^{54}$

\section{Acknowledgements}

The authors thank Jeannine Coburn for her assistance with cell culture as well as Veronica Ranner for her assistance with Bombyx mori photo-imaging. We would like to acknowledge funding from the Tissue Engineering Resource Center (TERC) (NIH EB002520) from the National Institute of Biomedical Imaging and Bioengineering, from the American Society of Biochemistry and Molecular Biology (ASBMB) (PROLAB Program), as well as from the National Council of Research and Technology from Argentina (CONICET).

\section{References}

1 P. A. Heidenreich, J. G. Trogdon, O. A. Khavjou, J. Butler, K. Dracup, M. D. Ezekowitz, E. A. Finkelstein, Y. Hong, S. C. Johnston, A. Khera, D. M. Lloyd-Jones, S. A. Nelson, G. Nichol, D. Orenstein, P. W. F. Wilson and Y. J. Woo, Circulation, 2011, 123, 933.

2 W. Wu, R. A. Allen and Y. Wang, Nat. Med., 2012, 18(7), 1148-1154.

3 X. Zhang, M. R. Reagan and D. L. Kaplan, Adv. Drug Delivery Rev., 2009, 61, 988.

4 V. Catto, S. Farè, G. Freddi and M. C. Tanzi, ISRN Vasc. Med., 2014, 2014, 1.

5 L. Gui and L. E. Niklason, Curr. Opin. Chem. Eng., 2014, 3, 68.

6 W. Zhang, L. S. Wray, J. Rnjak-Kovacina, L. Xu, D. Zou, S. Wang, M. Zhang, J. Dong, G. Li, D. L. Kaplan and X. Jiang, Biomaterials, 2015, 56, 68.

7 Á. E. Mercado-Pagán, Y. Kang, M. W. Findlay and Y. Yang, Mater. Sci. Eng., C, 2015, 49, 541.

8 H. Ahn, Y. M. Ju, H. Takahashi, D. F. Williams, J. J. Yoo, S. J. Lee, T. Okano and A. Atala, Acta Biomater., 2015, 16, 14.

9 T. Watanabe, K. Kanda, H. Ishibashi-Ueda, H. Yaku and Y. Nakayama, J. Artif. Organs, 2007, 10, 10.

10 C. Mandrycky, Z. Wang, K. Kim and D.-H. Kim, Biotechnol. Adv., 2016, 34, 422.

11 A. Shafiee and A. Atala, Trends Mol. Med., 2016, 22, 254.

12 S. Patra and V. Young, Cell Biochem. Biophys., 2016, 74, 93.

13 S. V. Murphy and A. Atala, Nat. Biotechnol., 2014, 32, 773.

14 I. T. Ozbolat and Y. Yu, IEEE Trans. Biomed. Eng., 2013, 60, 691.
15 M. S. Baguneid, A. M. Seifalian, H. J. Salacinski, D. Murray, G. Hamilton and M. G. Walker, Br. J. Surg., 2006, 93, 282.

16 E. C. Novosel, C. Kleinhans and P. J. Kluger, Adv. Drug Delivery Rev., 2011, 4, 300.

17 J. Lanza, R. Langer and R. Vacanti, Principle of Tissue Engineering, Academic Press in an Imprint of Elsevier, Oxford, 4th edn, 2007, vol. 3, p. 1936.

18 B. Kundu, R. Rajkhowa, S. C. Kundu and X. Wang, Adv. Drug Delivery Rev., 2013, 4, 457.

19 C. Correia, S. Bhumiratana and L. Yan, Acta Biomater., 2012, 8, 2483.

20 N. Kasoju and U. Bora, Adv. Healthcare Mater., 2012, 4, 393.

21 G. H. Altman, F. Diaz, C. Jakuba, T. Calabro, R. L. Horan, J. Chen, H. Lu, J. Richmond and D. L. Kaplan, Biomaterials, 2003, 24, 401.

22 Y. Zhang, W. Fan, Z. Ma, C. Wu, W. Fang, G. Liu and Y. Xiao, Acta Biomater., 2010, 6, 3021.

23 Y. Wang, E. Bella, C. S. D. Lee, C. Migliaresi, L. Pelcastre, Z. Schwartz, B. D. Boyan and A. Motta, The synergistic effects of 3-D porous silk fibroin matrix scaffold properties and hydrodynamic environment in cartilage tissue regeneration, Biomaterials, 2010, 31, 4672.

24 S. Huang and X. Fu, J. Controlled Release, 2010, 142, 149.

25 A. J. Engler, S. Sen, H. L. Sweeney and D. E. Discher, Cell, 2016, 4, 677.

26 S. Liu, C. Dong, G. Lu, Q. Lu, Z. Li, D. L. Kaplan and H. Zhu, Acta Biomater., 2013, 9, 8991.

27 X. Wang, X. Zhang, J. Castellot, I. Herman, M. Iafrati and D. L. Kaplan, Biomaterials, 2008, 29, 894.

28 T. Yucel, M. L. Lovett and D. L. Kaplan, J. Controlled Release, 2014, 190, 381.

29 M. Lovett, C. Cannizzaro, L. Daheron, B. Messmer, G. VunjakNovakovic and D. L. Kaplan, Biomaterials, 2007, 28, 5271.

30 H. Liu, X. Li, G. Zhou, H. Fan and Y. Fan, Biomaterials, 2011, 32, 3784 .

31 L. Soffer, X. Wang, X. Zhang, J. Kluge, L. Dorfmann, D. L. Kaplan and G. Leisk, J. Biomater. Sci., Polym. Ed., 2008, 19, 653.

32 C. Zhang, D. Song, Q. Lu, X. Hu, D. L. Kaplan and H. Zhu, Biomacromolecules, 2012, 13, 2148.

33 Q. Lv, C. Cao and H. Zhu, Polym. Int., 2005, 54, 1076.

34 Q. Lu, X. Wang, S. Lu, M. Li, D. L. Kaplan and H. Zhu, Biomaterials, 2011, 32, 1059.

35 D. Yao, S. Dong, Q. Lu, X. Hu, D. L. Kaplan, B. Zhang and H. Zhu, Biomacromolecules, 2012, 13, 3723.

36 M. L. Lovett, C. M. Cannizzaro, G. Vunjak-Novakovic and D. L. Kaplan, Biomaterials, 2008, 29, 4650.

37 X. Wang, H. J. Kim, P. Xu, A. Matsumoto and D. L. Kaplan, Langmuir, 2005, 21, 11335.

38 W. S. Rasband, ImageJ, U. S. National Institutes of Health, Bethesda, Maryland, USA, 1997-2016, http://imagej.nih.gov/ij/. 39 X. Hu, D. L. Kaplan and P. Cebe, Macromolecules, 2006, 39, 6161. 40 C. Vepari and D. Kaplan, Prog. Polym. Sci., 2007, 32, 991.

41 J. H. Kang, J. M. Gimble and D. Kaplan, Tissue Eng., Part A, 2009, 15, 2227.

42 J. Brown, C.-L. Lu, J. Coburn and D. L. Kaplan, Acta Biomater., 2015, 11, 212. 
43 H.-J. Jin, J. Park, R. Valluzzi, P. Cebe and D. L. Kaplan, Biomacromolecules, 2004, 5, 711.

44 H. Anwarul, M. Adnan, A. Nasim, H. Monowar, P. Arghya, R. D. Mehmet, D. Fariba and K. Ali, Acta Biomater., 2014, 1, 11.

45 M. Lovett, G. Eng, J. a. Kluge, C. Cannizzaro, G. Vunjaknovakovic and D. L. Kaplan, Tubular silk sca olds for small diameter vascular grafts, Organogenesis, 2010, 6, 217.

46 C. Wang, L. Cen, S. Yin, Q. Liu, W. Liu, Y. Cao and L. Cui, Biomaterials, 2010, 31, 661.

47 M. Stekelenburg, M. C. M. Rutten, L. H. E. H. Snoeckx and F. P. T. Baaijens, Tissue Eng., Part A, 2009, 15, 1081.

48 N. L'Heureux, S. Paquet, R. Labbe, L. Germain and F. A. Auger, FASEB J., 1998, 12, 47.
49 A. Karimi, M. Navidbakhsh, A. Shojaei and S. Faghihi, Mater. Sci. Eng., C, 2013, 33, 2550.

50 V. Catto, S. Farè, I. Cattaneo, M. Figliuzzi, A. Alessandrino, G. Freddi, A. Remuzzi and M. C. Tanzi, Mater. Sci. Eng., C, 2015, 54, 101.

51 L. S. Wray, X. Hu, J. Gallego, I. Georgakoudi, F. G. Omenetto, D. Schmidt and D. L. Kaplan, J. Biomed. Mater. Res., Part B, 2011, 99, 89.

52 A. de Mel, G. Punshon, B. Ramesh, S. Sarkar, A. Darbyshire, G. Hamilton and A. M. Seifalian, Bio-Med. Mater. Eng., 2009, 19, 317.

53 S. Sarkar, G. Y. Lee, J. Y. Wong and T. A. Desai, Biomaterials, 2006, 27, 4775.

54 N. C. Rivron, J. Liu, J. Rouwkema, J. de Boer and C. A. van Blitterswijk, Eur. Cells Mater., 2008, 15, 27. 